\title{
Incorporating Stress Estimation into User-centred Agent-based Platforms
}

\author{
Paulo Novais, Davide Carneiro, José Neves \\ DI-CCTC \\ University of Minho \\ Largo do Paço, 4704-553 Braga, Portugal \\ \{ pjon; dcarneiro; jneves\}@di.uminho.pt
}

\begin{abstract}
Current virtual environments for communication, cooperation and problem solving lack the rich context information that is present in face-to-face interactions. People rely on this context information, that includes body language or level of stress just to name a few, to take decisions. In this paper we present an approach whose objective is to be able to acquire context information about the users of such technological tools, making that information available for the tool and eventually to other people. We present a prototype being developed in the context of an agent-based conflict resolution platform. As a result, we achieve communication and problem solving virtual environments that are richer and closer to traditional environments, allowing people and software agents to take better and more rational decisions.
\end{abstract}

Keywords: Multi-agent systems, Online Dispute Resolution, Stress.

\section{Introduction}

Agent-based approaches are nowadays a popular way of implementing a wide variety of technological tools for problem-solving [1]. This will most likely continue to be true as future trends point out to multi-agent systems as a the way to enable next generation computing, not by means of very powerful computers but by potentiating the power of communication between many independent components [2]. One of the fields that can definitely profit from such supporting frameworks is the one of Online Dispute Resolution [3]. Online Dispute Resolution is now seen as the new technology-based paradigm for solving disagreements, replacing litigation in court. However, as the human's role gradually loses its substance as the main decision maker, some elements must be taken into consideration, so that conflict resolution processes guided by autonomous software agents will incorporate the best facets of the human experts. In fact, some potential threats that this technology may present have to be pointed out [4], namely the loss of important context information (e.g. body language [5], level of stress, emotional responses).

In this paper we support the idea that these issues should be taken into consideration when developing agent-based applications, making them usercentric. Specifically, we present an approach merging insights from Multi-Agent Systems (MAS) and Ambient Intelligence (AmI) [6]. Specifically, in this paper we focus on developing stress-aware [7] conflict resolution platforms. 


\section{Main Purpose}

We are extending the agent-based UMCourt conflict resolution platform, being developed under the TIARAC funded project [8] with two new agents that provide an estimation of the level of stress of the users in real time: (1) Stress Manager receives information from Stress Sensors and estimates a value of stress for a given user and (2) Stress Sensor - multiple instances of this agent exist, one for each different source of information about stress. Stress Sensors register with a Stress Manager.

The Stress Manager is responsible for receiving information from the Stress Sensors and computing an estimation of the level of stress. Five different stress sensors have been developed. The Personal Conflict deduces stress information from the interaction of the parties, by analyzing the utility of the proposals they create and how they react to each proposal they receive (e.g. accept, reject, reply). In a few words, specific conflict resolution styles are associated to different levels of stress. This sensor is detailed in [9].

The four remaining sensors are incorporated in the mobile devices used as interfaces, which are equipped with touch screens and accelerometers. The following information can be extracted from this hardware: (1) Acceleration - the value of the acceleration on the handheld device can be correlated with the level of stress: stressed users move more and in more abrupt ways; (2) Touch pattern stressed users evidence touch patterns that are different from calm users; (3) Accuracy - is a measure of touches on active controls (e.g. buttons, sliders) versus on passive areas (e.g. areas with disabled or no controls) and (4) Intensity - the intensity of the touch varies depending on the level of stress (e.g. stressed users tend to touch the screen with more intensity);

\section{Demonstration}

Our approach consists in analyzing such information about each user in order to study its evolution. This stream of information is transmitted to the Stress Manager by each Stress Sensor, so that it can be analyzed and translated into a value that stands for the user stress, useful to the whole agent-platform. The contribution of each source of stress to the overall value of stress at time $t$ is defined as depicted in formula 1. In order to shape the stress temporal evolution, the up to date values have a weight greater than the older ones. This may be configured by changing the value of parameter $b$, i.e., the higher the value, the faster the weight of the older ones decays.

Evidently, in no case two individuals are affected by stress in the same way. Thus, the approach presented includes a training phase and an operational phase. The training phase includes the touch events outside the scope of the conflict resolution, typically while the user is performing less stressful tasks (e.g., managing personal information, consulting past cases). During this phase the information collected is used to establish what can be considered the normal state of stress of the party. During the operational phase, the information on the stress is 
compared with the value obtained from the training phase in order to characterize in a more accurate form the level of stress. This is essential once what we consider as a normal level of stress changes from person to person.

$$
\text { contribution }_{s, t}=W_{s} * \sum_{i=0}^{i=t-1} \frac{b^{i}}{\sum_{j=0}^{j=t-1} b^{j}} * v_{i, s}
$$

Where,

contribution $_{s, t}$ denotes contribution of sensor $s$ at time $t$;

$W_{s}$ denotes the weight of the sensor $s$;

$v_{i, s}$ denotes list of values of the sensor $s$;

We now present some examples of the kind of information that the proposed agents can provide. Figure 1a depicts how the variation of the touch intensity is approached by a quadratic function whose parameters are then input to a previously trained J48 classifier, allowing to classify each touch as stressed or not. Figure 1b shows different touch patterns for calm users (orange dashed line) and stressed ones (blue line).

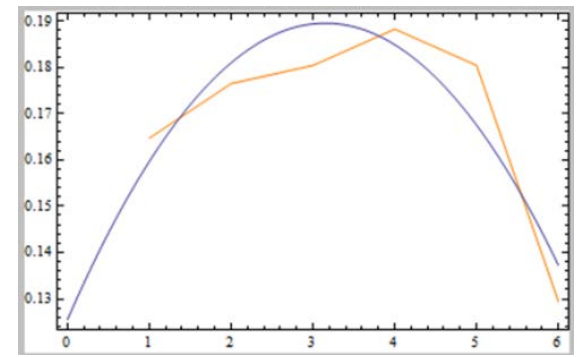

(a)

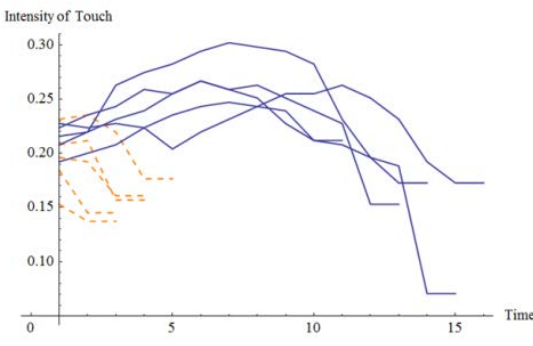

(b)

Fig. 1. Studying touch patterns through approximations to quadratic functions (a); different touch patterns of calm users (orange dashed line) and stressed users (blue line) (b).

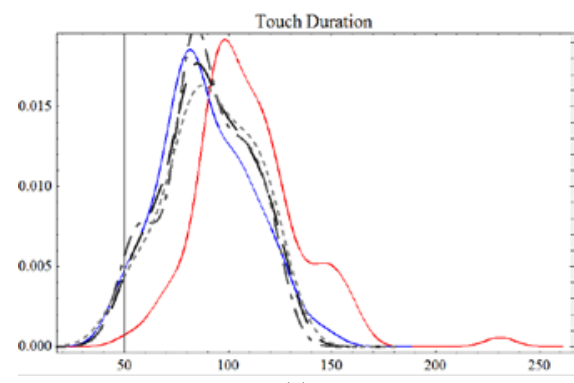

(a)

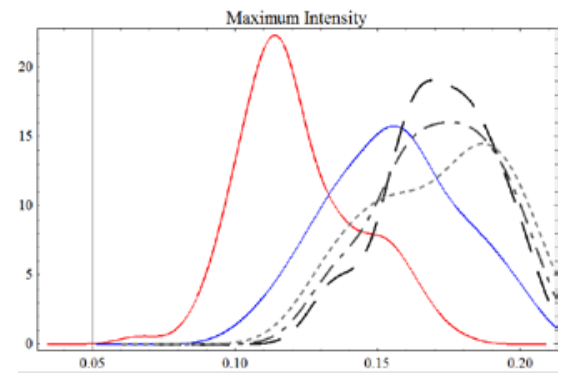

(b)

Fig. 2. How touch duration varies with stress for a given user (a); how touch intensity varies with stress for a given user (b).

Figure 2a and $2 \mathrm{~b}$ are histograms depicting significant differences in touch patterns for a user in different levels of stress. In these figures, the red line represents data collected without stress, the blue line represents all the data with 
stress and black lines with smaller values of dashing represent increased levels of stress. It is possible to see in Figure 2a that increased levels of stress result in smaller durations of touch. On the other hand, Figure $2 b$ shows that increased levels of stress are associated to an increased maximum value of the touch intensity.

\section{Conclusions}

The humanistic side is commonly left aside in virtual environments. Consequently, there is the risk of excluding important context information that is present in face-to-face interactions and that we rely on to take decisions. The approach presented has as main objective to enrich virtual environments with access to this context information. This information can then be used by either the platform or even a mediator that is conducting the process, to perceive how each issue or event is affecting each party and take better and justified decisions.

Acknowledgments. The work described in this paper is included in TIARAC Telematics and Artificial Intelligence in Alternative Conflict Resolution Project (PTDC/JUR/71354/2006), which is a research project supported by FCT (Science \& Technology Foundation), Portugal. The work of Davide Carneiro is also supported by a doctoral grant by FCT (SFRH/BD/64890/2009).

\section{References}

2. Luck, M., McBurney, P., Shehory, O., Willmott, S., Agent Technology: Computing as Interaction (A Roadmap for Agent Based Computing), AgentLink (2005)

3. Katsh, E., Rifkin, J., Online dispute resolution - resolving conflicts in cyberspace. Jossey-Bass Wiley Company, San Francisco (2001)

4. Larson, D.: Technology Mediated Dispute Resolution. Proceeding of the 2007 conference on Legal Knowledge and Information Systems: JURIX 2007: The Twentieth Annual Conference. IOS Press Amsterdam, The Netherlands (2007)

5. Mehrabian, A.: Silent Messages - A Wealth of Information about Nonverbal Communication. Personality \& Emotion Tests \& Software. Los Angeles (2009)

6. Carneiro, D., Novais, P., Costa, R., Neves, J., Developing Intelligent Environments with OSGi and JADE, Artificial Intelligence in Theory and Practice III, Bramer M. (Ed), IFIP AI, Springer (2010)

7. Jones, F., Kinman, G.: Approaches to Studying Stress. In F. Jones \& J. Bright (Eds.), Stress: Myth, Theory and Research. Harlow: Pearson Education (2001)

8. Carneiro, D., Novais, P., Neves, J., An Agent-based Architecture for Multifaceted Online Dispute Resolution Tools, in Developing Concepts in Applied Intelligence, Mehrotra K., Mohan C., Oh J., Varshney P., Ali M. (eds), Springer - Studies in Computational Intelligence, ISBN: 978-3-642-21331-1, pp. 89-94 (2011)

9. Carneiro, D., Gomes, M., Novais, P., Andrade, F., Neves, J., Automatic Classification of Personal Conflict Styles in Conflict Resolution, Frontiers in Artificial Intelligence and Applications, IOS Press, Proceedings of the $24^{\text {th }}$ International Conference on Legal Knowledge and Information Systems (2011) 